\title{
Characterization of New Transcripts Enriched in the Mouse Retina and Identification of Candidate Retinal Disease Genes
}

\author{
Julie Lord-Grignon, ${ }^{1}$ Nicolas Tétreault, ${ }^{1}$ Alan J. Mears, ${ }^{2}$ Anand Swaroop, ${ }^{2,3}$ and \\ Gilbert Bernier ${ }^{1,4}$
}

Purpose. Most retinal disease genes are preferentially expressed in photoreceptors, the light-sensitive cells involved in phototransduction. In addition, some of the genes linked to retinal diseases are essential for normal retinal development. The goal of this study was to identify new transcripts enriched in photoreceptors involved in retinal development or diseases.

Methods. To isolate uncharacterized retinal transcripts, the bioinformatic method Digital Differential Display (DDD) was used. RNA in situ hybridization was used to characterize geneexpression patterns.

Results. Twenty-seven mouse ESTs highly represented in retinal libraries were identified. Eight ESTs were predominantly expressed in photoreceptors and/or in the retinal pigment epithelium (RPE), whereas transcripts for other ESTs were detected more ubiquitously in the retinal cells or abundantly in ganglion cells and/or the inner nuclear layer. Mapping of the corresponding human orthologues of the photoreceptor/RPEenriched genes revealed that two of them are candidate disease genes for retinitis pigmentosa, loci RP22 and RP28. Both of these are predominantly expressed in rod photoreceptors. The candidate RP22 gene codes for a putative transmembrane protein showing homology to $\mathrm{Cln} 8$ (ceroid lipofuscinosis, neuronal 8), in which gene mutations are associated with photoreceptors degeneration in mice. Also identified were two genes expressed in photoreceptors that are candidate disease genes for recessive Bardet-Biedl syndrome type 3 (BBS3) and recessive ataxia with RP (AXPC1).

Conclusions. This study demonstrates how bioinformatic analysis can be used to identify novel tissue-specific genes relevant to development and diseases. (Invest Ophthalmol Vis Sci. 2004;45:3313-3319) DOI:10.1167/iovs.03-1350

From the ${ }^{1}$ Maisonneuve-Rosemont Hospital, Montreal, Quebec, Canada; the ${ }^{4}$ Department of Ophthalmology, University of Montreal, Montreal, Quebec, Canada; and the Departments of ${ }^{2} \mathrm{Ophthalmology}$ and Visual Sciences and ${ }^{3}$ Human Genetics, Kellogg Eye Center, University of Michigan, Ann Arbor, Michigan.

Supported by grants from the Fonds de Recherche en Ophthalmologie de l'Université de Montréal (FROUM), the Conseil de Recherches en Sciences Naturelles et en Génie du Canada (CRSNG), National Institutes of Health (EY11115), The Foundation Fighting Blindness, and Research to Prevent Blindness (RPB). AS is a recipient of the Lew R. Wasserman Merit Award from RPB. GB is supported by the Fonds de la Recherche en Santé du Québec (FRSQ).

Submitted for publication December 14, 2003; revised March 29, 2004; accepted May 5, 2004.

Disclosure: J. Lord-Grignon, None; N. Tétreault, None; A.J. Mears, None; A. Swaroop, None; G. Bernier, None

The publication costs of this article were defrayed in part by page charge payment. This article must therefore be marked "advertise$m e n t "$ in accordance with 18 U.S.C. $\$ 1734$ solely to indicate this fact.

Corresponding author: Gilbert Bernier, Maisonneuve-Rosemont Hospital, 5415 Boulevard l'Assomption, Montreal, Quebec H1T 2M4, Canada; gbernier.hmr@ssss.gouv.qc.ca.
$R$ etinal degenerative diseases affect millions of people worldwide and constitute a group of highly heterogeneous diseases. To date, 163 independent loci have been linked to human retinal diseases, and more than half of the disease-associated genes have been identified (http://www. sph.uth.tmc.edu/retnet/disease.htm; RetNet is provided in the public domain by the University of Texas Houston Health Science Center, Houston, TX). Photoreceptors, the specialized cells involved in light detection and phototransduction, represent the main genetic target of several retinopathies, such as retinitis pigmentosa, macular degeneration, Leber congenital amaurosis, and cone-rod dystrophy. ${ }^{1-4}$ In addition, because photoreceptor metabolism and physical integrity are highly dependent on direct interactions with the retinal pigment epithelium (RPE), genetic defects affecting RPE viability or functions can lead to photoreceptors degeneration. ${ }^{5}$

During development, the six neuronal and one glial cell types present in the retina are generated in a conserved sequential order. During this process, both extrinsic and intrinsic cues control the number and identity of the generated cell types. $^{6-8}$ Genetic studies have revealed that development of the photoreceptors is in part governed by the combinatorial action of two transcription factors, Crx and Nrl. Crx encodes an Otx-like homeobox protein and is expressed in both cones and rods. ${ }^{9,10}$ Crx directly regulates the transcription of photoreceptor-specific genes such as rhodopsin, cone opsin(s), and arrestin, and genetic ablation of $C r x$ in mice results in abnormal development of photoreceptors and absence of phototransduction. ${ }^{9-11}$ In contrast, $\mathrm{Nrl}$ is specifically expressed in rod and not cone photoreceptors and can act synergistically with Crx to regulate the transcription of rhodopsin and other rod genes. $^{9,12-14}$ Targeted deletion of $\mathrm{Nrl}$ in mice results in the complete absence of rods and an enhanced S-cone function, suggesting a functional transformation of rods into $S$ cones. ${ }^{15}$ Notably, missense mutations in human $N R L$ have been associated with autosomal dominant retinitis pigmentosa (RP), and mutations in $C R X$ have been associated with cone-rod dystrophy, RP, and Leber congenital amaurosis, highlighting their crucial functions in both normal development and disease. ${ }^{16-20}$

In addition to $N R L$ and $C R X$, several photoreceptor- and RPE-enriched genes (e.g., rhodopsin) have been associated with retinal and macular degenerative diseases. ${ }^{4}$ Several research groups have used molecular methods (such as subtraction cloning and expressed sequence tag [EST] sequencing) and bioinformatics strategies to isolate novel genes that are enriched in photoreceptors and RPE. ${ }^{21-31}$ Herein, we describe the isolation of novel retina-enriched genes that were identified using a bioinformatics method. Of the 27 ESTs analyzed, 8 are highly enriched in photoreceptors and/or RPE cells, and human orthologues of 4 are located within cytogenetic intervals of uncloned retinal disease loci. 
TABLE 1. Identification of Known Genes Involved in Retinal Development and Diseases using DDD

\begin{tabular}{|c|c|c|c|c|}
\hline Mouse Gene & DDD Results & Protein Function & Linked Human Eye Disease & Null Phenotype in Mice \\
\hline Rom 1 & $0 \times-108 \times$ & Glycoprotein & & Photoreceptor degeneration \\
\hline $\mathrm{Nrl}$ & $0 \times-74 \times$ & Transcription factor & Dominant retinitis pigmentosa & No rod photoreceptors \\
\hline $\operatorname{Cr} x$ & $0 \times-71 \times$ & Transcription factor & Cone-rod dystrophy, Leber congenital amaurosis & No phototransduction/blind \\
\hline Nr2e3 & $0 \times-71 \times$ & Nuclear receptor & Enhanced S cone syndrome & \\
\hline$R x$ & $0 \times-53 \times$ & Transcription factor & & Anophthalmia \\
\hline Tubby/TULP1 & $0 \times-50 \times$ & Unknown & Retinitis pigmentosa & Retinal degeneration \\
\hline Rpgrip1 & $0 \times-45 \times$ & Unknown & Retinitis pigmentosa & \\
\hline Hes 5 & $1 \times-42 \times$ & Transcription factor & & Reduce number of Müller glial cells \\
\hline Pax6 & $1 \times-37 \times$ & Transcription factor & Aniridia/anophthalmia & Small eye/anophthalmia \\
\hline$R s 1 b$ & $1 \times-37 \times$ & Cell adhesion & Retinitis pigmentosa & \\
\hline Lhx 2 & $1 \times-34 \times$ & Transcription factor & & Anophthalmia \\
\hline Hes2/Matb3 & $1 \times-34 \times$ & Transcription factor & & No bipolar cells \\
\hline Brn-3.2/POU4 & $0 \times-32 \times$ & Transcription factor & & No ganglion cells \\
\hline$T l x$ & $0 \times-32 \times$ & Nuclear receptor & & Ganglion cells degeneration \\
\hline Matb5 & $0 \times-21 \times$ & Transcription factor & & No ganglion cells \\
\hline Chx10 & $1 \times-18 \times$ & Transcription factor & Microphthalmia & Microphthalmia \\
\hline
\end{tabular}

Differential expression levels of known retinal genes as obtained by DDD. The numbers represent the frequency of the individual ESTs among pool A (non-neuronal libraries, left) compare with pool B (eye and retinal libraries, right). Most depicted mouse genes have been involved in normal eye development and/or retinal cells maintenance, whereas mutations of several human orthologues are linked to human retinopathies.

\section{Methods}

\section{RT-PCR Analysis and cDNA Cloning}

Primers design for RT-PCR amplification of the EST cDNA was performed using software from the Virtual Genome Center (http://alces. med.umn.edu/rawprimer.html; maintained in the public domain). All amplification products were generated using total RNA (TRIzol; Invitrogen-Gibco, Burlington, Ontario, Canada) and a one-step RT-PCR amplification kit (Invitrogen-Gibco). Amplification products corresponding to the predicted size were directly cloned into the PCR2.0 vector (Invitrogen-Gibco), analyzed by restriction enzyme mapping and sequenced. The human cDNA used for in situ hybridization (Image Clone ID: 6172676; GenBank ID: BU195682; http://www.ncbi.nlm. nih.gov/Genbank; provided in the public domain by the National Center for Biotechnology Information [NCBI], Bethesda, MD) is homologue to the reference sequence AW964851 and was obtained from the American Type Culture Collection (ATCC; Manassas, VA). The cDNA was originally sequenced from a human retinoblastoma cDNA library and has a length of 909 bp (cloned in the pCMV-SPORT6 vector; Invitrogen-Gibco).

\section{Nrl Knockout Mice and Real-Time PCR Analysis}

Animals used in the study were maintained according to the ARVO Statement for the Use of Animals in Ophthalmic and Vision Research. Retinas were dissected from 20-week-old $\mathrm{Nrl}^{-\prime-}$ mice and age- and background-matched wild-type control animals. Total RNA was isolated from the retina (TRIzol; Invitrogen-Gibco) and was reverse transcribed (Superscript II; Invitrogen-Gibco) according to the manufacturer's guidelines. Quantitative real-time PCR was performed (I-cycler with Optical Module; BioRad, Hercules, CA; using Sybr Green I; Molecular Probes, Eugene, OR). Reactions were performed in triplicate and the average multiple of change ( $x$-fold) between knockout and wild-type samples calculated after normalization to Hprt.

\section{In Situ Hybridization}

Linearized plasmid DNA $(1 \mu \mathrm{g})$ was used to produce sense and antisense digoxigenin (DIG)-labeled RNA probes (Roche Diagnostics, Montreal, Quebec, Canada) using either T7 or T3 RNA polymerase (Invitrogen-Gibco) for every EST clone. Riboprobes were purified by ethanol precipitation, resuspended in TE buffer and filtered (ProbeQuant G-50 Micro Column; Amersham Biosciences, Arlington Heights, IL). Adult mice from the albinos CD1 strain (Charles River, St. Constant, Quebec, Canada) were euthanatized in a $\mathrm{CO}_{2}$ chamber according to local
Animal Protection Comity (APC) guidelines. Eyes were enucleated and embedded in optimal cutting temperature compound (OCT, Tissue Tek; Sakura Finetek, Torrance, CA) and snap frozen in liquid nitrogen. Eye sections were cut at $8 \mu \mathrm{m}$ using a cryostat (Leica, Heidelberg, Germany) and air dried on coated glass slides (Super-Frost Plus; Fisher Scientific, Montreal, Quebec, Canada). Human donor retinas were obtained from the eye bank of Maisonneuve-Rosemont Hospital, according to the hospital ethics committee and the Declaration of Helsinki guidelines for the use of human tissue in research. The following steps of the RNA in situ hybridization procedure were performed according to a published protocol. ${ }^{28}$ Developed sections were observed by microscope (DMRE; Leica), and data were acquired with a digital camera (Retiga EX; QIMAGING, Canada; with OpenLab, ver. 3.1.1 software; OpenLab, Toronto, Ontario, Canada).

\section{Results}

To identify novel transcripts enriched in the retina, we used Digital Differential Display (DDD), a computational method (UniGene; http://www.ncbi.nlm.nih.gov/unigene; provided in the public domain by the National Center for Biotechnology Information, Bethesda, MD) that allows comparative analysis of the frequency of individual ESTs among pools of cDNA libraries. This method has been used to isolate novel genes involved in cardiac muscle development in mice. ${ }^{32}$ We chose to compare a pool of 303,773 ESTs (pool A) present in non-neuronal libraries (e.g., liver, kidney, lung) with 37,851 ESTs (pool B) present in whole-eye and retinal libraries. Whole-eye libraries were included in pool B to increase the overall number of eye-enriched ESTs. Removal of nonretinal transcripts, specific for the lens, for example, was achieved later by the analysis of the EST content within each cluster. To estimate the validity of the assay, we first analyzed the differential representation of known and abundant eye-specific transcripts, such as for rhodopsin (photoreceptors) and crystallin (high expression in lens). The rhodopsin transcript was present 1 time in pool $\mathrm{A}$ (non-neuronal), whereas it was present 1054 times in pool B (eye and retina), and the crystallin $\alpha 1$ transcript was absent from pool A, whereas it was present 225 times in pool B. Second, ubiquitous housekeeping transcripts (e.g., the intermediate filament protein vimentin or $\beta$-actin), were not selected as differentially represented. Third, several genes known to be involved in either mouse eye development or human retinal diseases were selected as highly differentially repre- 
TABLE 2. Expression Pattern Analysis of 27 Genes Enriched in Eye and Retinal Libraries

\begin{tabular}{|c|c|c|c|c|c|c|c|c|}
\hline \multirow[b]{2}{*}{ Clone } & \multirow[b]{2}{*}{$\begin{array}{c}\text { GenBank } \\
\text { Accession No. }\end{array}$} & \multirow[b]{2}{*}{$\begin{array}{l}\text { DDD } \\
\text { Results }\end{array}$} & \multicolumn{4}{|c|}{ RT-PCR } & \multirow[b]{2}{*}{$\begin{array}{l}\text { Retinal Expression } \\
\text { (RNA In Situ) }\end{array}$} & \multirow[b]{2}{*}{$\begin{array}{c}\text { Expression Levels in } \\
\mathrm{Nrl}^{-\prime-} \text { Versus WT Retina }\end{array}$} \\
\hline & & & ov e10.5 & e10.5 ov- & ad Liver & ad Retina & & \\
\hline 1 & AK053683 & $0 \times-50 \times$ & + & + & - & + & Ubiquitous & N/A \\
\hline 2 & CA879414 & $0 \times-50 \times$ & - & - & - & + & RPE & Reduced $4.8 \times$ \\
\hline 3 & BQ930206 & $0 \times-45 \times$ & - & - & - & ++ & Ubiquitous & Increased $1.6 \times$ \\
\hline 4 & NP_080044 & $2 \times-45 \times$ & - & - & - & ++ & $\mathrm{PR} / \mathrm{IS}+\mathrm{RPE}>\mathrm{INL}$ & Reduced $2.8 \times$ \\
\hline 5 & XM_354606 & $0 \times-40 \times$ & N/A & N/A & N/A & N/A & N/A & N/A \\
\hline 6 & XM_133706 & $0 \times-40 \times$ & - & - & - & ++ & PR/IS/OS & Reduced $7.1 \times$ \\
\hline 7 & AK053799 & $0 \times-37 \times$ & N/A & N/A & N/A & N/A & N/A & N/A \\
\hline 8 & BY721394 & $0 \times-34 \times$ & - & - & - & + & RPE + IS > INL & Reduced $2.3 \times$ \\
\hline 9 & ВC049556 & $0 \times-34 \times$ & + & + & + & + & INL & N/A \\
\hline 10 & AW490610 & $0 \times-34 \times$ & + & + & - & + & Ubiquitous & N/A \\
\hline 11 & AK045045 & $0 \times-32 \times$ & - & + & + & ++ & PR/IS > INL & Increased $3.9 \times$ \\
\hline 12 & AK044366 & $0 \times-18 \times$ & ++ & + & - & ++ & INL & N/A \\
\hline 13 & AK046050 & $0 \times-82 \times$ & N/A & N/A & N/A & N/A & N/A & N/A \\
\hline 14 & AK0444422 & $0 \times-42 \times$ & + & + & - & + & $\mathrm{PR}>\mathrm{INL}$ & Reduced $1.4 \times$ \\
\hline 15 & AK040168 & $0 \times-34 \times$ & + & + & + & + & Ubiquitous & N/A \\
\hline 16 & BC031985 & $0 \times-34 \times$ & + & + & - & + & $\mathrm{GC}+\mathrm{HC}$ & N/A \\
\hline 17 & AK015515 & $0 \times-32 \times$ & + & + & + & + & Ubiquitous & N/A \\
\hline 18 & AK035621 & $0 \times-32 \times$ & - & - & - & + & $\mathrm{RPE}>\mathrm{INL}$ & N/A \\
\hline 19 & AK011438 & $1 \times-29 \times$ & + & + & + & + & Ubiquitous & N/A \\
\hline 20 & XM_358363 & $1 \times-24 \times$ & + & + & - & + & $\mathrm{RPE}+\mathrm{INL}+\mathrm{GC}$ & N/A \\
\hline 21 & NM_146223 & $3 \times-34 \times$ & - & + & - & ++ & Ubiquitous & N/A \\
\hline 22 & AK039726 & $1 \times-32 \times$ & - & + & + & + & N/A & N/A \\
\hline 23 & AK044733 & $0 \times-31 \times$ & - & + & - & + & Ubiquitous & N/A \\
\hline 24 & AK044313 & $0 \times-27 \times$ & - & - & - & + & $\mathrm{PR}>\mathrm{INL}$ & Reduced $8.0 \times$ \\
\hline 25 & NM_011881* & $0 \times-27 \times$ & - & - & - & ++ & PR/IS & Reduced $3.0 \times$ \\
\hline 26 & AK044611 & $0 \times-26 \times$ & - & - & - & + & N/A & N/A \\
\hline 27 & AK044330 & $0 \times-21 \times$ & + & + & - & + & Ubiquitous & N/A \\
\hline
\end{tabular}

Individual EST sequences were amplified by RT-PCR on RNA samples from e10.5 mouse optic vesicle (ov), whole e10.5 embryo minus the optic vesicle (ov-), adult (ad) liver and retina. The retinal expression pattern is based on RNA in situ hybridization performed on adult mouse eye sections.

* Rhodopsin kinase (Rhok). RPE, retinal pigment epithelium; PR, photoreceptor; IS, inner segment; OS, outer segment; INL, inner nuclear layer; GC, ganglion cells; HC, horizontal cells; and N/A, not available.

sented (Table 1). This included the transcription factors Pax6, Chx10, Crx, and Nrl, which are essential for normal retinal development and/or maintenance in both humans and mice. ${ }^{11,15-19,33-36}$

Approximately 70 potentially novel EST sequences were differentially represented at least 20 times more in pool B than in pool A. We analyzed these sequences by BLAST against the mouse and human EST databases. Known gene sequences (published and characterized) or ESTs that were not highly represented in retinal libraries (i.e., $<75 \%$ of the total number of ESTs present in one cluster) were excluded from our study. This proportion of $75 \%$ was chosen arbitrarily, to eliminate genes that were not truly enriched in the retina, such as genes abundant in the brain, lens, cornea, or eye mesenchyme. This analysis identified 27 potentially novel EST sequences highly represented in the retina (Table 2).

\section{Expression Pattern Analysis of the 27 Retina-Enriched ESTs}

The cDNAs for the 27 selected ESTs were cloned by reverse transcription-polymerase chain reaction (RT-PCR), analyzed by restriction enzyme digestion, and sequenced. Three ESTs were not amplified by RT-PCR, despite numerous attempts (Table 2). RT-PCR was also used to analyze the gross expression profile of the ESTs at e10.5 (eye and whole embryo minus the eye) and in adult (neural retina and liver). Ten ESTs (c2, c3, c4, c6, c8, c11, c18, c21, c24, and c25) were predominantly expressed in the adult neural retina by RT-PCR (Table 2). By in situ hybridization on adult mouse eye sections, transcripts for six of these clones were detected abundantly in the outer nuclear layer (ONL) of the retina (containing the cell body of photoreceptors), whereas three additional clones were predominantly expressed in the RPE (Figs. 1A-I). From the 10 clones enriched in the retina as detected by RT-PCR, only clone 3 displayed a ubiquitous expression pattern in the retina (Table 2). Additional in situ hybridization results with the remaining clones revealed that three ESTs (c9, c12, and c20) were expressed predominantly in the inner nuclear layer (INL) of the retina (containing amacrine, bipolar, horizontal, and Müller glial cells), whereas another EST (c16) was strongly expressed in ganglion cells (Figs. 1J-M). The remaining EST clones were either expressed at low levels or showed ubiquitous expression in the retina. As negative and positive controls, we used sense (Fig. 1N) and antisense (Fig. 1O) probes corresponding to the mouse Pax6 cDNA. As expected, we observed specific expression of Pax 6 in amacrine (INL) and ganglion cells. ${ }^{37} \mathrm{On}$ sequence analysis, clone 25 was found to correspond to the well-known rhodopsin kinase ( $R b o k$ ) gene. $R b o k$ is specifically expressed in both cone and rod photoreceptors and was used as the positive control for expression in photoreceptors (Fig. 1E). Corresponding sense riboprobes were used as the negative control for all ESTs that were analyzed by in situ hybridization (not shown).

Detailed expression analysis of the photoreceptors/RPEenriched transcripts revealed that clone 6 was highly and preferentially expressed in the photoreceptors (Fig. 1A), whereas clones $4,8,11,14$, and 24 were also expressed in the inner nuclear layer. Clones 4, 6, and 11, and to a lesser extent clone 8 , also showed expression in photoreceptor inner segments. Clones 2, 4, 8, and 18 also were expressed in the RPE (Fig. 1). 

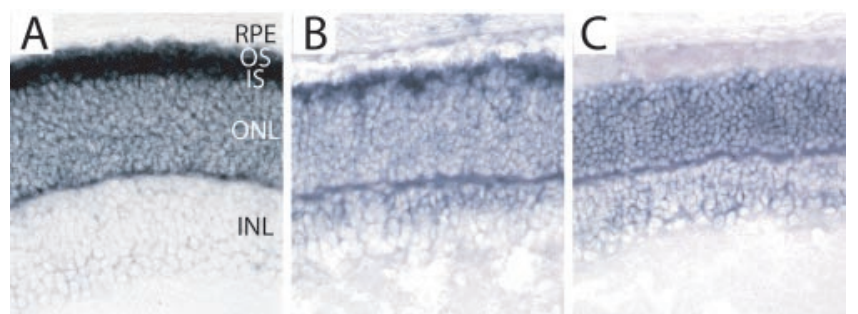

C6

GC
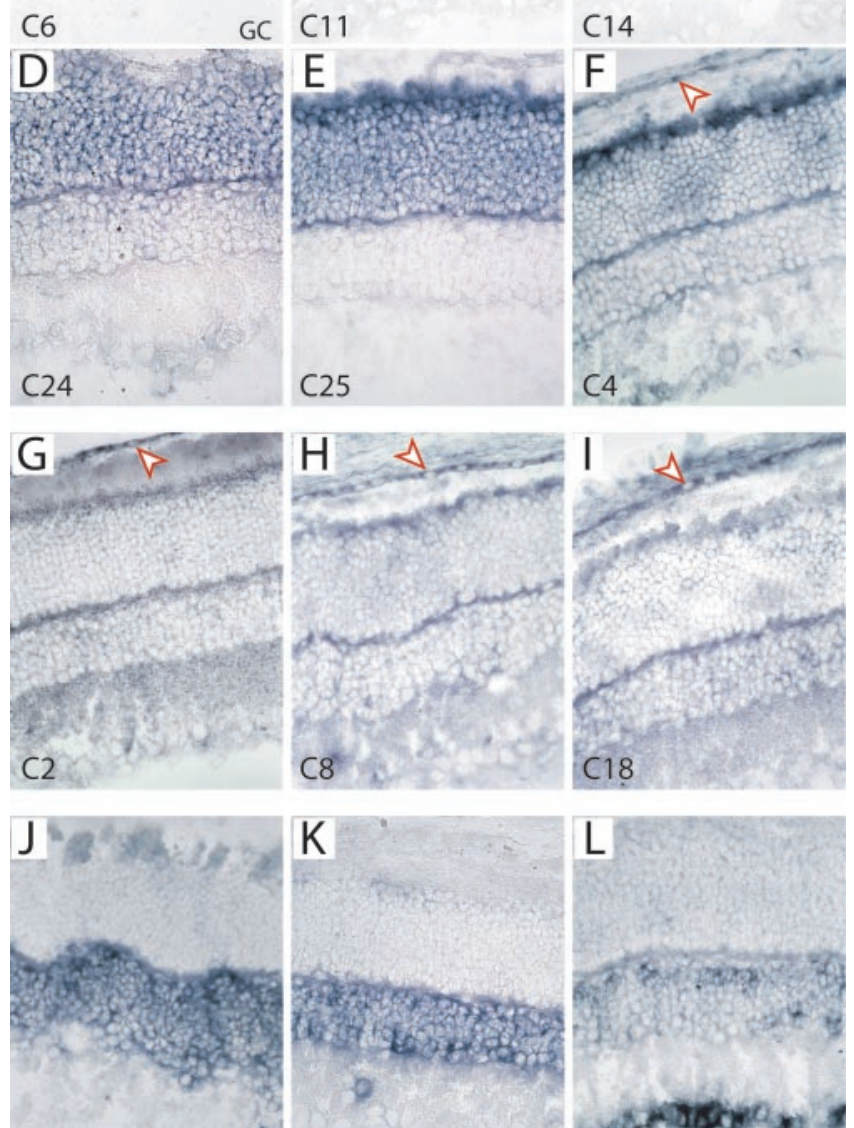

C9
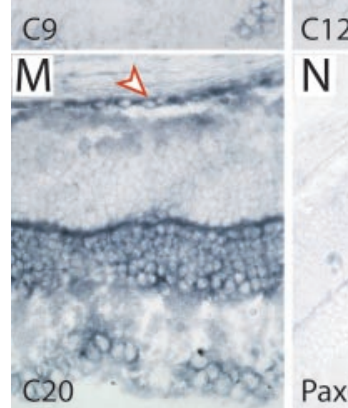

$\mathrm{N}$
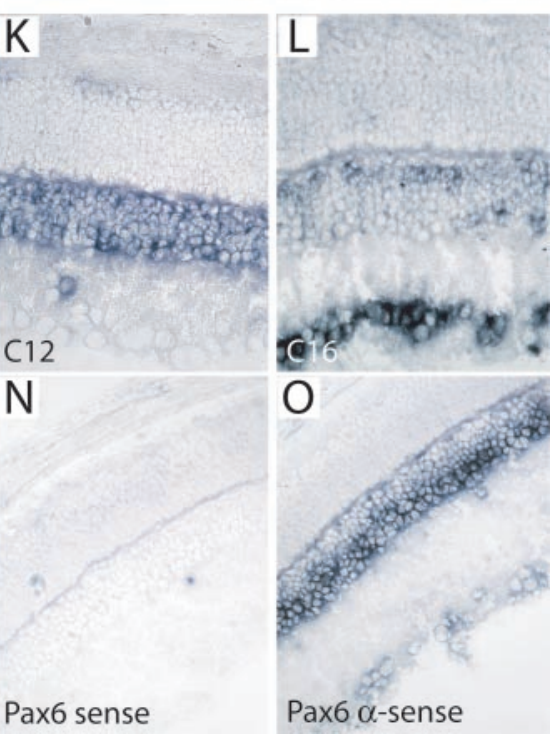

FIGURE 1. Cellular expression pattern of novel retina-enriched genes. Nonradioactive RNA in situ hybridization on albino adult mouse eye sections showed that six ESTs are abundantly expressed in the cellular layer that contains photoreceptors (A- F). In addition, three ESTs (GI) are enriched in the RPE (arrowbead) and in photoreceptors (H). Some of the retina-enriched genes are specific for cell types other than photoreceptors (J-M). Pax 6 sense $(\mathbf{N})$ and antisense $(\mathbf{O})$ probes were used as negative and positive controls, respectively. RPE, retinal pigment epithelium; OS, outer segment; IS, inner segment; ONL, outer nuclear layer; INL, inner nuclear layer; GC, ganglion cell layer.

\section{Expression of Photoreceptors and RPE Enriched Transcripts in Nrl Mutant Retina}

The outer nuclear layer contains both rod and cone (S and $\mathrm{M}$ types) photoreceptors. In the $\mathrm{Nrl}$ knockout mice $\left(\mathrm{Nrl}^{-{ }^{-}}\right)$, functionally and morphologically distinct rods were not present; instead, there was an increase in the S-cones. ${ }^{15}$ This apparent functional transformation of rods into S-cones resulted in a reduction in rod-enriched transcripts and an increase in cone-specific transcripts. To investigate whether our photoreceptor-enriched ESTs are expressed predominantly in rods or cones (or both), we compared their expression levels by quantitative real-time PCR (qRT-PCR) using the retinal RNA from the wild-type and $\mathrm{Nrl}$ knockout mice (Table 2). We hypothesize that some of the genes showing lower expression levels in $\mathrm{Nrl}^{-/-}$retina are associated with rod function. However, many genes showing altered expression could reflect secondary changes due to the loss of Nrl function and/or remodeling of retinal organization in the $\mathrm{Nrl}^{-/-}$retina. Of the nine EST sequences examined, only one (c11) showed a high increase in expression levels in the $\mathrm{Nrl}^{-/-}$retina, whereas expression levels of six ESTs were reduced in the $\mathrm{Nrl}^{-1-}$ retina. Notably, clones 6 and 24 displayed a seven- to eightfold reduction in their expression in the $\mathrm{Nrl}^{-/-}$retina (Table 2).

\section{Identification of Human Orthologues and Candidate Retinal Disease Genes}

To investigate whether the ONL/RPE-enriched ESTs we identified were candidate genes for a human retinal disease, we used the public human EST database to find putative human orthologues. The human orthologues for six mouse ESTs and their location on human chromosomes were readily identified by this analysis. For two remaining clones that had no identifiable human gene/EST orthologue (c14 and c24), we searched the human genome sequence database by BLAST for significant homology using the mouse EST sequences. To enhance the odds of identifying human genomic intervals for all ESTs we searched for cDNA homology against the mouse genome and used both $5^{\prime}$ and $3^{\prime}$ flanking mouse genomic regions $(\sim 40 \mathrm{~kb})$ for BLAST search against the human genome. This resulted in a highly significant match at 8p22-p23 for clone 14 and in possible match at $2 \mathrm{p} 13$ for clone 24 . Mapping of the putative human orthologue of clone 24 at $2 \mathrm{p} 13$ was further confirmed by mouse-human chromosome synteny analysis using the Celera Discovery System (http://www.celeradiscoverysystem. com; Celera, Rockville, MD). In mice, clone 24 is located on chromosome 6 at position $82,685 \mathrm{M}$, close to Amsh at 82,524 $\mathrm{M}$, and human $A M S H$ is located at $2 \mathrm{p} 12$.

We succeeded in the identification and mapping of a corresponding human orthologue for the eight photoreceptors/ RPE- enriched genes. Four of them were located within cytogenetic intervals for uncloned retinal disease genes (Table 3), whereas two mapped at loci for already cloned disease genes (clone 4 at 13q34 and clone 18 at 19q13.3) and two at a cytogenetic interval where there is no linked retinal disease (clone 14 at 8p22-8p23 and clone 2 at 9q22). The four candidate disease genes we found are: clone 6 (Homo sapiens; AW964851) at 16p12.1 for recessive RP (RP22), clone 8 (Homo sapiens; BG699018) at 3p11-p12 for recessive BardetBiedl syndrome (BBS3), clone 11 (Homo sapiens; AL627109) at 1q32.1-32.2 for recessive ataxia with RP (AXPC1) and clone 24 (Homo sapiens; Hs2_22340) at 2p13 for recessive RP (RP28). ${ }^{38-41}$ We also attempted to identify and map the human orthologues for the remaining ESTs showing specific expression in the retina (Figs. 1J-M) but not enriched in photoreceptors (i.e., c9, c12, c16, and c20). Orthologous human genes for clone 9 and 12 could not be identified. However, clone 16 human orthologue (Homo sapiens; Q15825) mapped at 
TABLE 3. Chromosomal Mapping and Identification of Candidate Retinal Disease Genes

\begin{tabular}{|c|c|c|c|c|c|}
\hline Clone & $\begin{array}{c}\text { GenBank } \\
\text { Accession No. }\end{array}$ & $\begin{array}{c}\text { Protein Homology or } \\
\text { Function }\end{array}$ & $\begin{array}{l}\text { Acc. No. Human } \\
\text { Orthologue }\end{array}$ & $\begin{array}{l}\text { Chromosomal } \\
\text { Mapping }\end{array}$ & Linked Retinal Disease \\
\hline 6 & XM_133706 & Transmembrane protein & AW964851 & $16 \mathrm{p} 12.1$ & Recessive rp (RP22)-uncloned gene \\
\hline 8 & BY721394 & Nectin-like protein & BG699018 & 3p11-p12 & Recessive Bardet-Bidl syndrome (BBS3)-uncloned gene \\
\hline 11 & AK045045 & Unknown & AL627109 & $1 \mathrm{q} 32.1-\mathrm{q} 32.2$ & Recessive ataxia with rp (AXPC1)-uncloned gene \\
\hline 24 & AK044313 & Unknown & Hs2_22340 & $2 \mathrm{p} 13$ & Recessive rp (RP28) (2p11-p16)-uncloned gene \\
\hline
\end{tabular}

ESTs from UniGene clusters were BLAST against the human EST, NR or genome databases to identify human orthologues. Genes enriched in photoreceptors, and/or in the RPE and which the human orthologues map to a retinal disease loci for which the gene has not been cloned are showed. rp, retinitis pigmentosa.

$8 \mathrm{p} 11.1$ in the cytogenetic interval for recessive cone-rod dystrophy (CORD9) (http://www.sph.uth.tmc.edu/retnet/disease. htm; RetNet). Clone 16 codes for a nicotinic and cholinergic $\alpha$-receptor (Cntnap2) protein but represents an unlikely candidate disease gene for CORD9, due to its predominant expression in ganglion cells. Clone 20 codes for a contactin associated protein-like 2 (Cntnap2) and its human orthologue (Homo sapiens; Q9UHC6) maps at 7q35-q36, where there are no linked retinal diseases.

To test the hypothesis that these human transcripts are true orthologues of the identified mouse ESTs, we analyzed the expression of the human orthologue of clone 6 (AW964851) in the mature human retina. By in situ hybridization on human retinal sections, we observed strong expression of AW964851 in the cell body of photoreceptors (ONL) and in the photoreceptors inner segment (Figs. 2A, 2B). This is very similar to the in situ hybridization results obtained with clone 6 on retinal sections from adult mice (Fig. 1A).

\section{Comparative Analysis with Previous Studies}

Several groups have reported on the identification and mapping of ESTs enriched for expression in the retina. ${ }^{21-31} \mathrm{We}$ retrospectively compared our results with those of all these previous studies to evaluate whether the four genes we herein identified represent true novel candidate retinal disease genes (Table 3). Globally, only one of the candidate retinal disease gene presented herein (clone 11) was identified in a previously published study. ${ }^{28}$ Thus, the human orthologues of the three remaining ESTs identified here represent true novel candidate retinal disease genes at three independent loci (Table 3).

\section{Discussion}

Using the DDD method, we identified yet uncharacterized ESTs that are enriched in retina/eye cDNA libraries. Of these, 12 ESTs demonstrate a cell-type-specific mRNA distribution within the retina, with eight showing predominant expression in photoreceptors and/or RPE. Human orthologues for four
photoreceptors/RPE-enriched ESTs mapped within distinct cytogenetic intervals containing uncloned retinal disease genes.

\section{Gene-Expression Pattern and Diseases Phenotype}

Retinitis pigmentosa (RP) is primarily characterized by night blindness, constriction of visual field, and retinal dystrophy, without symptoms outside the visual system. Consequently, expression of most cloned RP genes is in fact highly enriched in photoreceptors, although there are some notable exceptions (e.g., RPGR for X-linked RP). ${ }^{4,42}$ The two candidate disease genes for RP22 and RP28 that we identified were found to be expressed almost uniquely in the retina and were highly enriched in photoreceptors (Fig. 1). In addition, these two clones show the most altered expression levels in the $\mathrm{Nrl}^{-{ }^{-}}$retina. This is noteworthy, because $N R L$ mutations have been linked to dominant RP in humans. ${ }^{19}$ We also showed that the putative human orthologue of clone 6 is highly expressed in human photoreceptors, supporting the hypothesis that this represents a true orthologue. Therefore, these two genes fulfill all criteria to be considered valid candidate genes for RP22 and RP28.

In this study, we identified a candidate disease gene (clone 11) for posterior column ataxia with retinitis pigmentosa (AXPC1). AXPC1 is characterized by visual impairment, proprioceptive loss, sensory ataxia, and areflexia. ${ }^{41}$ Based on this phenotype, one can predict predominant gene expression in photoreceptors, muscle spindles, and/or Ia afferent sensory fibers of the dorsal root ganglia and cerebellum. Analysis of the UniGene cluster for clone 11 showed that its expression extends outside the retina in organs such as brain, olfactory mucosa, and skin, making clone 11 a good candidate disease gene for AXPC1.

We identified a candidate disease gene (clone 8) for recessive Bardet-Biedl syndrome (BBS3) at 3p11-p12. BBS3 is a rare, multisystemic autosomal recessive disease characterized by retinal dystrophy, renal abnormalities, obesity, dysmorphic extremities, and hypogenitalism in males. ${ }^{38}$ The gross expression pattern of clone 8 suggests predominant expression in the

Figure 2. A candidate disease gene for RP22 is expressed in human photoreceptors. Nonradioactive RNA in situ hybridization on adult human retina showing high expression of the human orthologue (AW964851) of murine clone 6 in the outer nuclear layer (ONL) and in the inner segment of photoreceptors (A, B). No specific signal was observed with the corresponding sense probe $(\mathbf{C})$. INL, inner nuclear layer; GC, ganglion cell layer. Original magnification: $(\mathbf{A}, \mathbf{C}) \times 200$; (B) $\times 630$.

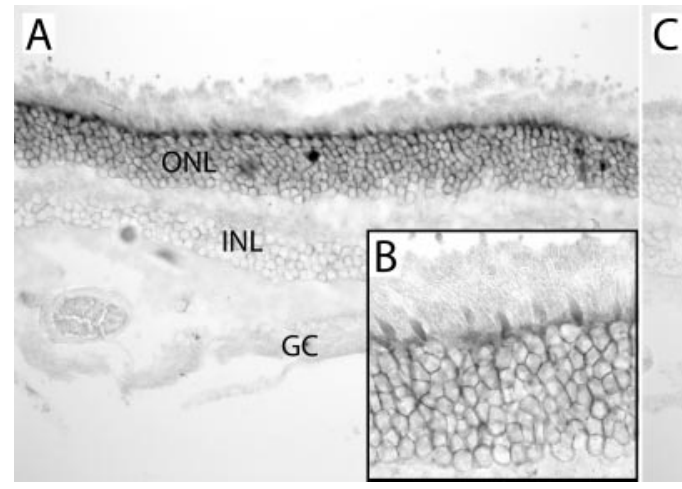

ONL

INL

GC 
retina, making this EST an unlikely candidate gene for BBS3. However, UniGene cluster analysis (BY721394/Mm.197421) showed that clone 8 is expressed in organs other than the eye, including brain, spinal cord, testis, spleen and mammary gland. Thus, more extensive studies, such as linkage and mutational analyses, are needed to exclude this as a candidate disease gene for BBS3.

\section{Predicted Cellular and Molecular Function of the Identified Candidate Retinal Disease Genes}

We identified four candidate retinal disease genes at four independent loci. Using the predicted translational product of these transcripts and BLAST, we compared the amino-acid sequence of these putative proteins with the public protein databases (NCBI). Of these four genes, two encode protein motifs showing homology with known proteins (Table 3). Clone 6 human orthologue (Homo sapiens; AW964851) encodes for a putative protein with a central TLC homology domain containing five transmembrane $\alpha$-helices (GenBank accession no. NP_113666). Overall, the predicted translation product of this gene is very well conserved in vertebrates, showing $\sim 94 \%$ amino-acid identity with the mouse (Mus musculus; XP_133706) and $\sim 70 \%$ amino-acid identity with zebrafish (Danio rerio; AAH59567). We also found a putative protein in Drosophila (Drosophila melanogaster; AF181646) that shows $\sim 34 \%$ identity with human NP_113666. A sequence comparison with other proteins revealed that NP_113666 possesses $\sim 49 \%$ identity and $\sim 67 \%$ similarity with CT120 and $\sim 28 \%$ identity and $\sim 50 \%$ similarity with CLN8. CT120 encodes a membrane-associated protein that is conserved in different organisms, including plants, and it appears to be involved in amino-acid transport and glutathione metabolism. ${ }^{43}$ CLN8 encodes a transmembrane protein and is the gene mutated in the mouse mutant motor neuron ( $m n d$ ) degeneration and in humans with the autosomal recessive disorder progressive epilepsy with mental retardation (EPMR; Mendelian Inheritance in Man [MIM] 600143). ${ }^{44}$ These diseases are part of the neuronal ceroid lipofuscinoses, a group of neurodegenerative disorders characterized by the accumulation of autofluorescent lipopigment in various tissues. Notably, mnd mice display photoreceptors degeneration whereas another lipofuscinoses-related gene, $C L N 3$, is the mutated gene in Batten-disease, a degenerative disorder characterized by earlyonset retinal pigmentary degeneration and later mental deterioration. ${ }^{45,46}$ Coincidentally, the CLN3 gene is located at 16p11.2, thus relatively close to AW964851 at 16p12.1 (http:// www.sph.uth.tmc.edu/retnet/disease.htm; RetNet). The other candidate disease gene we identified and which displays homology with known genes is clone 8 . Clone 8 is weakly homologous ( $52 \%$ of homology) to nectin-like 1 (necl-1). Necl are a recently characterized group of proteins that share structural similarities with nectins. Nectins are a family of $\mathrm{Ca}^{2+}$ independent immunoglobulin-like cell-cell adhesion molecules involved in the formation of cadherin-based adherens junctions. ${ }^{47}$ In turn, Necls heterophilically interact with nectins to regulate cell polarization and migration. Thus, clone 8 could be involved in cell-cell interactions between the RPE and photoreceptors to either stabilize or organize adherens junctions.

In conclusion, the gene discovery method we used complements the large genomic scale analysis (e.g., SAGE or microarrays) and is efficient for small-scale hypothesis-driven studies. The use of UniGene EST database and the DDD software therefore represents a rapid, inexpensive, and efficient method for identifying novel tissue-specific transcripts relevant to normal development and diseases.

\section{References}

1. Inglehearn CF. Molecular genetics of human retinal dystrophies. Eye. 1998;12:571-579.

2. Rattner A, Sun H, Nathans J. Molecular genetics of human retinal disease. Annu Rev Genet. 1999;33:89-131.

3. Clarke G, Heon E, McInnes RR. Recent advances in the molecular basis of inherited photoreceptor degeneration. Clin Genet. 2000; 57:313-329.

4. Pacione LR, Szego MJ, Ikeda S, Nishina PM, McInnes RR. Progress toward understanding the genetic and biochemical mechanisms of inherited photoreceptor degenerations. Annu Rev Neurosci. 2003;26:657-700.

5. Kameya S, Hawes NL, Chang B, et al. Mfrp, a gene encoding a frizzled related protein, is mutated in the mouse retinal degeneration 6. Hum Mol Genet. 2002;11:1879-1886.

6. Levine EM, Fuhrmann S, Reh TA. Soluble factors and the development of rod photoreceptors. Cell Mol Life Sci. 2000;57:224-234.

7. Livesey FJ, Cepko CL. Vertebrate neural cell-fate determination: lessons from the retina. Nat Rev Neurosci. 2001;2:109-118.

8. Edlund T, Jessel TM. Progression from extrinsic to intrinsic signaling in cell fate specification: a view from the nervous system. Cell. 1999;96:211-224.

9. Chen S, Wang QL, Nie Z, et al. Crx, a novel Otx-like pairedhomeodomain protein, binds to and transactivates photoreceptor cell-specific genes. Neuron. 1997;19:1017-1030.

10. Furukawa T, Morrow EM, Cepko CL. Crx, a novel otx-like homeobox gene, shows photoreceptor-specific expression and regulates photoreceptor differentiation. Cell. 1997;91:531-541.

11. Furukawa T, Morrow EM, Li T, Davis FC, Cepko CL. Retinopathy and attenuated circadian entrainment in Crx-deficient mice. Nat Genet. 1999;23:466-470.

12. Swaroop A, Xu JZ, Pawar $\mathrm{H}$, et al. A conserved retina-specific gene encodes a basic motif/leucine zipper domain. Proc Natl Acad Sci USA. 1992;89:266-270.

13. Rehemtulla A, Warwar R, Kumar R, et al. The basic motif-leucine zipper transcription factor $\mathrm{Nrl}$ can positively regulate rhodopsin gene expression. Proc Natl Acad Sci USA. 1996;93:191-195.

14. Mitton KP, Swain PK, Chen S, et al. The leucine zipper of NRL interacts with the CRX homeodomain: a possible mechanism of transcriptional synergy in rhodopsin regulation. J Biol Chem. 2000;275:29794-29799.

15. Mears AJ, Kondo M, Swain KS, et al. Nrl is required for rod photoreceptor development. Nat Genet. 2001;29:447-452.

16. Swain PK, Chen S, Wang QL, et al. Mutations in the cone-rod homeobox gene are associated with the cone-rod dystrophy photoreceptor degeneration. Neuron. 1997;19:1329-1336.

17. Freund CL, Gregory-Evans CY, Furukawa T, et al. Cone-rod dystrophy due to mutations in a novel photoreceptor-specific homeobox gene (CRX) essential for maintenance of the photoreceptor. Cell. 1997;91:543-553.

18. Freund CL, Wang QL, Chen S, et al. De novo mutations in CRX homeobox gene associated with Leber congenital amaurosis. Nat Genet. 1998;18:311-312.

19. Bessant DA, Payne AM, Mitton KP, et al. A mutation in NRL is associated with autosomal dominant retinitis pigmentosa. Nat Genet. 1999;21:355-356.

20. Swaroop A, Wang QL, Wu W, et al. Leber congenital amaurosis caused by a homozygous mutation (R90W) in the homeodomain of the retinal transcription factor CRX: direct evidence for the involvement of CRX in the development of photoreceptor function. Hum Mol Genet. 1999;8:299-305.

21. Gieser L, Swaroop A. Expressed sequence tags and chromosomal localization of cDNA clones from a substracted retinal pigment epithelium library. Genomics. 1992;13:873-876.

22. Bascom RA, Schappert K, McInnes RR. Cloning of the human and murine ROM1 genes: genomic organization and sequence conservation. Hum Mol Genet. 1993;2:385-391.

23. Guillonneau X, Piriev NI, Danciger M, et al. A nonsense mutation in a novel gene is associated with retinitis pigmentosa in a family linked to the RP1 locus. Hum Mol Genet. 1999;8:1541-1546.

24. Sohocki MM, Malone KA, Sullivan LS, Daiger SP. Localization of retina/pineal-expressed sequences: identification of novel candi- 
date genes for inherited retinal disorders. Genomics. 1999;58:2933.

25. Malone KA, Sohocki MM, Sullivan LS, Daiger SP. Identifying and mapping novel retinal-expressed ESTs from humans. Mol Vis. 1999;5:1-5.

26. Sinha S, Sharma A, Agarwal N, Swaroop A, Yang-Feng TL. Expression profile and chromosomal location of cDNA clones, identified from an enriched adult retina library. Invest Ophthalmol Vis Sci. 2000; $41: 24-28$

27. Mu X, Zhao S, Pershad R, et al. Gene expression in the developing mouse retina by EST sequencing and microarray analysis. Nucleic Acids Res. 2001;29:4983-4993.

28. Blackshaw S, Fraioli RE, Furukawa T, Cepko LC. Comprehensive analysis of photoreceptor gene expression and the identification of candidate retinal disease genes. Cell. 2001;107:579-589.

29. Kennan A, Aherne A, Palfi A, et al. Identification of an IMPDH1 mutation in autosomal dominant retinitis pigmentosa (RP10) revealed following microarray analysis of transcripts derived from retinas of wild-type and Rho-/- mice. Hum Mol Genet. 2002;11: 547-558.

30. Katsanis N, Worley KC, Gonzalez G, Ansley SJ, Lupski JR. A computational/functional genomics approach for the enrichment of the retinal transcriptome and the identification of positional candidate retinopathy genes. Proc Natl Acad Sci USA. 2002;99: $14326-14331$.

31. Yu J, Farjo R, MacNee SP, et al. Annotation and analysis of 10,000 expressed sequences tags from developing mouse eye and adult retina. Genome Biol. 2003;4:R65.

32. Wang D, Chang PS, Wang Z, et al. Activation of cardiac gene expression by myocardin, a transcriptional cofactor for serum response factor. Cell. 2001;105:851-862.

33. Hill RE, Favor J, Hogan BL, et al. Mouse small eye results from mutations in a paired-like homeobox-containing gene. Nature. 1991;354:522-525.

34. Ton CC, Hirvonen H, Miwa H, et al. Positional cloning and characterization of a paired box- and homeobox-containing gene from the aniridia region. Cell. 1991;67:1059-1074.

35. Burmeister M, Novak J, Liang MY, et al. Ocular retardation mouse caused by Chx10 homeobox null allele: impaired retinal progenitor proliferation and bipolar cell differentiation. Nat Genet. 1996; 12:376-384.
36. Ferda Percin E, Ploder LA, Yu JJ, et al. Human microphthalmia associated with mutations in the retinal homeobox gene CHX10. Nat Genet. 2000;25:397-401.

37. Marquardt T, Ashery-Padan R, Andrejewski N, et al. Pax6 is required for the multipotent state of retinal progenitor cells. Cell. 2001; 105:43-55.

38. Young TL, Woods MO, Parfrey PS, et al. Canadian Bardet-Biedl syndrome family reduces the critical region of BBS3 (3p) and presents with a variable phenotype. Am J Med Genet. 1998;78: 461- 467.

39. Finckh U, Cu S, Kumaramanickavel G, et al. Homozygosity mapping of autosomal recessive retinitis pigmentosa locus (RP22) on chromosome 16p12.1-p12.3. Genomics. 1998;48:341-345.

40. Gu S, Kumaramanickavel G, Srikumari CR, Denton MJ, Gal A. Autosomal recessive retinitis pigmentosa locus RP28 maps between D2S1337 and D2S286 on chromosome 2p11-p15 in an indian family. J Med Genet. 1999;36:705-707.

41. Higgins JJ, Morton DH, Loveless JM. Posterior column ataxia with retinitis pigmentosa (AXPC1) maps to chromosome 1q31-q32. Neurology. 1999;52:146-150.

42. Meindl A, Dry K, Herrmann K, et al. A gene (RPGR) with homology to the RCC1 guanine nucleotide exchange factor is mutated in X-linked retinitis pigmentosa (RP3). Nat Genet. 1996;13:35-42.

43. He X, Di Y, Li J, et al. Molecular cloning and characterization of CT120, a novel membrane-associated protein involved in aminoacid transport and glutathione metabolism. Biochem Biophys Res Commun. 2002;297:528-536.

44. Ranta S, Zhang Y, Ross B, et al. The neuronal ceroid lipofuscinoses in human EPMR and mnd mutant mice are associated with mutations in CLN8. Nat Genet. 1999;23:233-236.

45. Chang B, Bronson RT, Hawes NL, et al. Retinal degeneration in motor neuron degeneration: a mouse model of ceroid lipofuscinosis. Invest Ophthalmol Vis Sci. 1994;35:1071-1076.

46. Cooper JD. Progress towards understanding the neurobiology of Batten disease or neurolipofuscinosis. Curr Opin Neurol. 2003;16: 121-128.

47. Takai Y, Irie K, Shimizu K, Sakisaka T, Ikeda W. Nectins and nectin-like molecules: roles in cell adhesion, migration and polarization. Cancer Sci. 2003;94:655-667. 